\title{
AORTIC ROOT RECONSTRUCTION WITH PRESERVATION OF NATIVE AORTIC VALVE AND SINUSES IN AORTIC ROOT DILATATION WITH AORTIC REGURGITATION
}

\author{
Jacques A. M. van Son, MD, PhD \\ Roberto Battellini, MD \\ Marco Mierzwa, MD \\ Thomas Walther, MD \\ Rüdiger Autschbach, MD, PhD \\ Friedrich W. Mohr, MD, PhD
}

Background: We assessed the results of a modified technique for aortic root reconstruction including preservation of the native aortic valve and sinuses. Methods: A modified technique for reconstruction of the aortic root was devised in which the native aortic sinuses are preserved and remodeled, the diameter of the sinotubular junction is reduced, the ventriculoaortic junction is reinforced with a Dacron prosthesis, and the coronary ostia are reimplanted. Since January 1995, this modified operative technique was performed in 13 patients with a mean age of $54 \pm 21$ years. The median grade of aortic regurgitation was 3; in 10 patients it was caused by dilatation of the sinotubular junction, and 3 had additional annuloaortic ectasia. Results: The aortic crossclamping time was $61 \pm 18$ minutes. In-hospital mortality was 2 of $13(15.3 \%)$ patients, both deaths being related to complications of aortic dissection. In 1 patient aortic regurgitation increased to grade 3 , necessitating aortic valve replacement. At a mean follow-up of 2.1 years, the remaining 10 patients had stable aortic valve function with a median grade of regurgitation of 1. The mean New York Heart Association functional class was 1.2. Conclusions: Aortic root reconstruction with preservation of the native aortic valve and sinuses allows symmetric reconstruction of the aortic sinuses and adaptation of the diameters of the sinotubular and ventriculoaortic junctions, thus optimizing aortic valve function. Moreover, it prevents contact of the aortic valve leaflets with the Dacron graft, which may enhance the durability of the repair. (J Thorac Cardiovasc Surg 1999;117:1151-6)
$\mathrm{D}$ ilatation of the aortic root resulting from longstanding aortic regurgitation (AR), often associated with overstretched aortic leaflets and a damaged fibrosa layer, usually necessitates composite replacement of the aortic valve and ascending aorta. ${ }^{1-4}$ In a minority of patients, however, the aortic leaflets are remarkably normal despite long-standing AR. In such a situation, reconstruction of the aortic root with preservation of the aortic leaflets and resection of the sinuses of Valsalva, as reported by David and coworkers ${ }^{5,6}$ and Sarsam and Yacoub, ${ }^{7}$ seem to be attractive alternative options to avoid composite replacement of the aortic valve and

From the Department of Cardiac Surgery, Herzzentrum, University of Leipzig, Leipzig, Germany.

Received for publication Oct 26, 1998; revisions requested Dec 9, 1998; revisions received Feb 23, 1999; accepted for publication Feb 26, 1999.

Address for reprints: Jacques A. M. van Son, MD, PhD, Herzzentrum, University of Leipzig, Russenstrasse 19, D-04289, Leipzig, Germany.

Copyright $\odot 1999$ by Mosby, Inc.

$0022-5223 / 99 \$ 8.00+0 \quad \mathbf{1 2 / 1 / 9 8 2 2 3}$ ascending aorta. However, one of the major concerns regarding these operations is related to the mechanism of closure of the aortic valve leaflets. In both operative techniques, although aortic valve leaflet abrasion has not been reported as yet, we have echocardiographically documented that in some cases the aortic valve leaflets during systole are in contact with the rough texture of the Dacron tubular graft. In an attempt to minimize the potential problem of leaflet damage, we have recently developed a modified technique of aortic root reconstruction in which the native sinuses of Valsalva are preserved and remodeled. This report describes our experience with this modified technique.

\section{Patients and methods}

The aortic root. The aortic root is a complex structure that is composed of 3 leaflets, 3 sinuses of Valsalva, and 3 interleaflet triangles. The leaflets are attached to the aortic wall in a semilunar fashion with the attachment of each leaflet rising to its zenith at the sinotubular junction and being at its nadir just below the ringlike junction of the aortic wall and the ventricle (anatomic ventriculoaortic junction). ${ }^{8-10}$ The base of the normal leaflet is approximately 1.5 times longer than is 
Table I. Clinical characteristics of the patients

\begin{tabular}{ll}
\hline No. of patients & 13 \\
Age (y) & 54.2 \\
Mean & $14-77$ \\
Range & 4 \\
Marfan syndrome & \\
Aortic dissection & 2 \\
$\quad$ Type A & 1 \\
Type B & 1 \\
Aortic arch aneurysm & \\
NYHA functional class & 5 \\
Class II & 6 \\
Class III & 2 \\
Class IV & \\
Diameter of aortic root (mm) & 58 \\
Mean & $49-78$ \\
Range & \\
Aortic regurgitation & 1 \\
Grade 1 & 5 \\
Grade 2 & 4 \\
Grade 3 & 3 \\
Grade 4 & 1 \\
Coronary artery disease & 1 \\
Mitral regurgitation & 1 \\
Tricuspid regurgitation & 1 \\
Ischemic VSD & \\
\hline NYHA New Yo Heit Assition &
\end{tabular}

NYHA, New York Heart Association; VSD, ventricular septal defect.

its free margin. ${ }^{11}$ This arrangement of the leaflets results in optimal coaptation of the aortic valve during diastole and ensures unobstructed flow of blood during systole. The aortic root has the shape of a truncated cone, the diameter of which at the level of the sinotubular junction is approximately $15 \%$ smaller than that at the anatomic ventriculoaortic junction (at aortic "anulus"). ${ }^{9,11-13}$ The height of the sinuses corresponds approximately to $60 \%$ to $70 \%$ of the diameter of the aortic anulus. ${ }^{11-13}$ The noncoronary sinus is slightly larger than the left and right sinuses. In the aortic root, the fibrous skeleton forms the posterior wall of the left ventricular outflow tract in the area where the aortic leaflets are in fibrous continuity with those of the mitral valve. The 2 ends of this area of fibrous continuity are thickened to form the left and right fibrous trigones. The right fibrous trigone is continuous with the membranous part of the septum, and the latter 2 structures form the central fibrous body through which the atrioventricular conduction tissue penetrates to pass from the muscular atrioventricular septum to the crest of the ventricular septum.

Isolated aortic root dilatation is a degenerative process that is localized in the aortic sinuses (predominantly the noncoronary sinus) and at the sinotubular junction. It is commonly related to increased age and systemic hypertension. Dilatation of the sinotubular junction prevents the leaflets from coapting, with consequent AR. The aortic anulus remains normal or dilates minimally. In annuloaortic ectasia the diameter of the aortic anulus increases out of proportion to the size of the aortic leaflets. ${ }^{6}$ The increase in annular diameter and dilatation of aortic sinuses occur along the fibrous components of
Table II. Operative data

\begin{tabular}{ll}
\hline Diameter of graft (mm) & 26 \\
Median & $22-30$ \\
Range & \\
Aortic crossclamping time (min) & 61 \\
$\quad$ Mean & $45-220$ \\
Range & \\
CPB time (min) & 98 \\
Mean & $61-450$ \\
Range & \\
Additional procedures & 1 \\
Replacement of aortic arch & 1 \\
Reconstruction of aortic arch & 1 \\
Replacement of aortic arch and descending aorta \\
Mitral valve reconstruction & 1 \\
Tricuspid valve reconstruction & 1 \\
CABG & 2 \\
Closure of ischemic VSD & 1 \\
\hline CABG, Coronary artery bypass grafting; CPB, cardiopulmonary bypass; VSD, \\
ventricular septal defect.
\end{tabular}

the left ventricular outflow tract. ${ }^{5}$ Eventually this results in dilatation of the sinotubular junction and AR. ${ }^{6}$

Clinical data. From January 1995 to July 1998, 13 patients (7 female and 6 male patients) with aneurysms of the ascending aorta and aortic root and associated AR underwent reconstruction of the aortic root with preservation of the native aortic valve and sinuses. The mean age of the patients was $54 \pm 21$ years (range 14 to 77 years). All patients had a preoperative Doppler echocardiographic study, which quantitated the AR from 0 (none or trace) to 4 (severe). The median grade of AR was 3 . In 10 patients the AR was primarily caused by dilatation of the sinotubular junction. Four patients had Marfan syndrome, 3 of whom had additional annuloaortic ectasia. Aortic dissection was present in 3 patients (type $\mathrm{A}$ in 2 patients and type B in 1). Tables I and II summarize the preoperative clinical data and the operative data, respectively. All patients had a Doppler echocardiographic study before discharge from the hospital, 2 to 3 months later, and annually thereafter.

Operative techniques. Cardiopulmonary bypass is established by cannulating the proximal aortic arch or the femoral artery and the right atrium. The systemic temperature is lowered to $32^{\circ} \mathrm{C}$ or to $24^{\circ} \mathrm{C}$ in patients with aortic root dilatation and associated aortic arch disease. After aortic crossclamping, continuous antegrade infusion of cold crystalloid cardioplegic solution is used for myocardial protection. The left ventricle is vented with a cannula inserted through the right superior pulmonary vein and mitral valve. The ascending aorta is transected just beyond the aneurysmal dilatation (Fig 1, A). The aortic root is inspected with particular attention directed toward the state of the leaflets and the degree of dilatation of the sinuses and the sinotubular and ventriculoaortic junctions. The leaflet heights (length from midportion of the free margin to midportion of attachment margin) are measured and averaged. The coronary ostia are excised with small buttons of aortic wall. If a coronary ostium (particularly the left one) is 

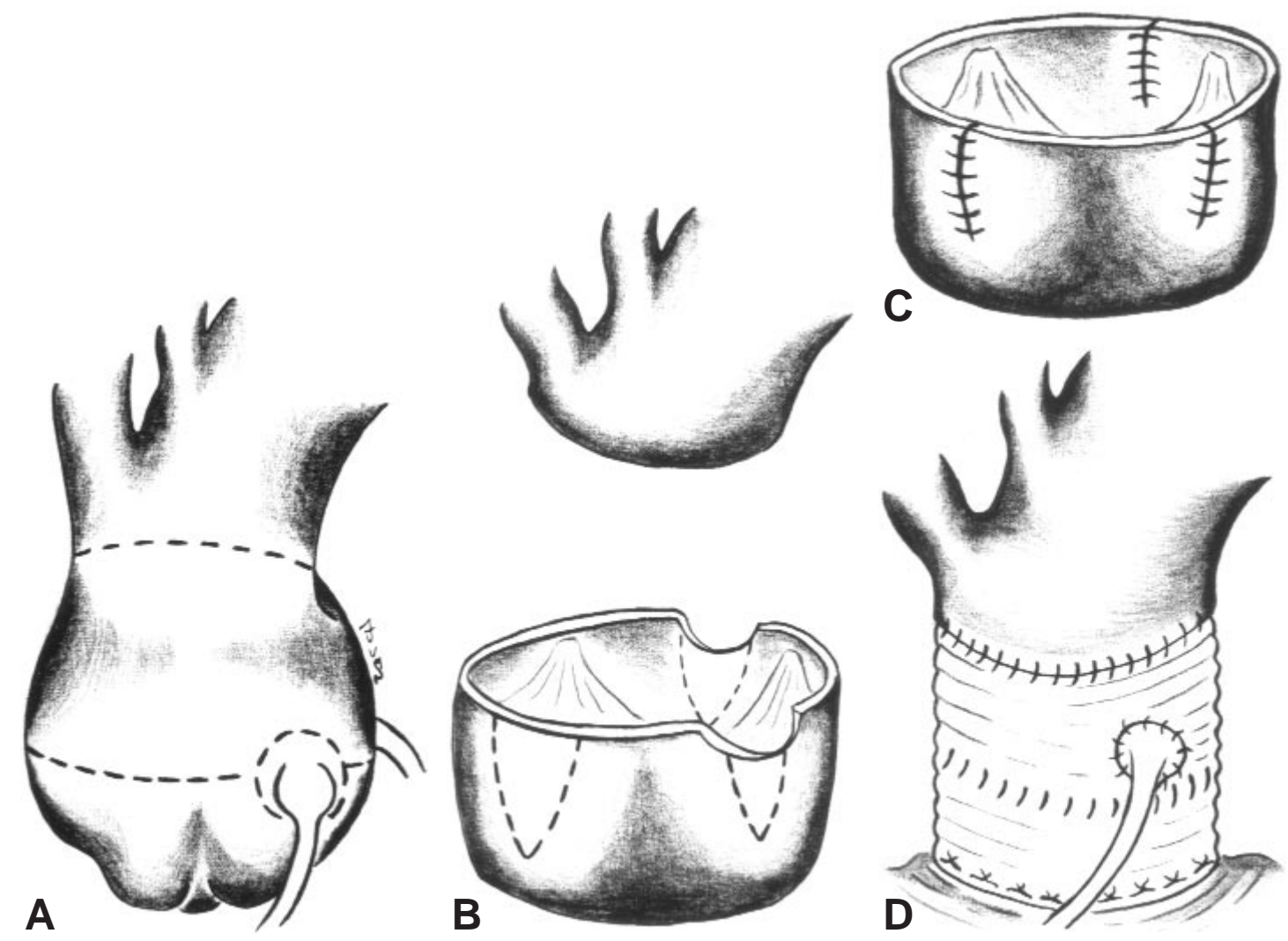

Fig 1. Technique of aortic root reconstruction with preservation of native aortic valve and sinuses and replacement of ascending aorta. A, The dashed lines indicate the incisions. The coronary ostia are excised with small buttons of aortic wall. B, Triangular pieces of dilated sinus wall are excised. The degree of excision varies with the redundancy of the sinus wall and usually is most extensive in the noncoronary sinus. $\mathbf{C}$, Reconstruction of the aortic root. D, Replacement of the ascending aorta with Dacron graft, which is secured to the aortic anulus with multiple interrupted sutures. The edge of the reconstructed aortic root is secured to the graft with a continuous suture. The coronary ostia are reimplanted.

in such proximity to the anulus that this prevents sufficient cephalad mobilization and reimplantation of the ostium (thus rendering reconstruction of that particular sinus with native tissue impossible), then that sinus may have to be resected and the aortic root remodeled according to the method of David and associates ${ }^{5,6}$ or Sarsam and Yacoub. ${ }^{7}$ From each sinus of Valsalva triangular pieces of dilated sinus wall are excised (Fig 1,B). The extent of the excision varies with the degree of dilatation of the sinuses and the sinotubular junction and is estimated as follows: first, the desired internal diameter of the ventriculoaortic junction is calculated by assuming that the internal radius of the ventriculoaortic junction is two thirds of the leaflet height. ${ }^{11-13}$ For instance, if the leaflet height is 15 $\mathrm{mm}$, the desired internal radius of the ventriculoaortic junction is $10 \mathrm{~mm}$ and the desired internal diameter of the ventriculoaortic junction thus equals $20 \mathrm{~mm}$. The desired internal circumference of the ventriculoaortic junction is 3.14 times the desired internal diameter, thus, in this case, $63 \mathrm{~mm}$. Assuming that the desired internal circumference of the aortic root is the same for the ventriculoaortic junction as for the sinotubular junction, the calculated internal circumference of the aortic root at the ventriculoaortic junction is subtracted from the internal circumference of the aortic root at the sinotubular junction. For instance, if the measured internal diameter of the sinotubular junction is $32 \mathrm{~mm}$, the internal circumference of the aorta at the sinotubular junction is 3.14 times $32 \mathrm{~mm}$, which equals $100 \mathrm{~mm}$. The length of redundant tissue is then $100 \mathrm{~mm}$ minus $63 \mathrm{~mm}$, which equals $37 \mathrm{~mm}$. Assuming that a rim of $3 \mathrm{~mm}$ of sinus wall is needed bilaterally for reconstruction of 1 excised sinus segment, approximately $6 \mathrm{~mm}$ of sinus wall is needed for reconstruction of 1 sinus, equaling 18 $\mathrm{mm}$ for 3 sinuses. The total amount of sinus wall that is to be excised in this example then is $37 \mathrm{~mm}$ minus $18 \mathrm{~mm}$, which equals $19 \mathrm{~mm}$. It is a matter of judgment as to how much tissue needs to be excised per sinus. Inasmuch as the aortic sinuses dilate, particularly in areas above the fibrous skeleton of the heart (noncoronary sinus and the medial halves of the right and left sinuses), in the majority of cases the excision is more extensive in the noncoronary sinus, followed by the right and left sinuses. 

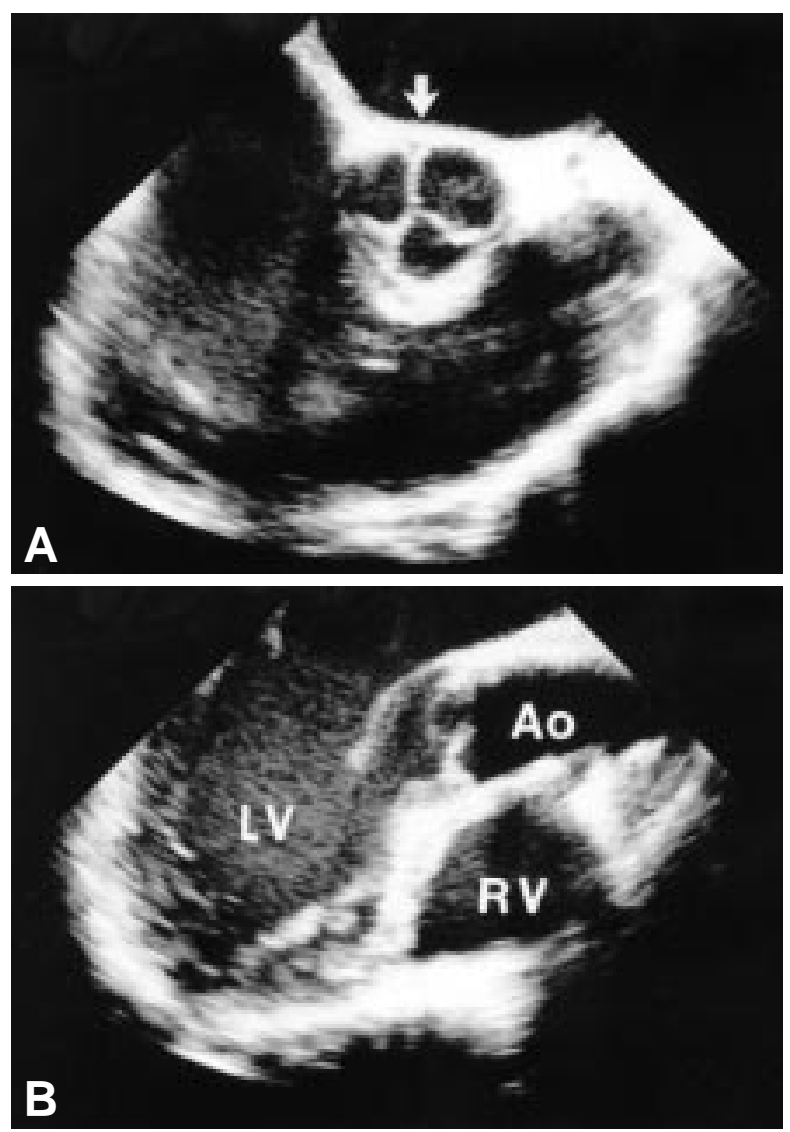

Fig 2. Transesophageal echocardiogram (transverse [A] and sagittal [B] views) of reconstructed aortic root with preservation of the native aortic valve and sinuses and replacement of the ascending aorta. Note the excellent coaptation of the aortic valve leaflets (arrow) and the symmetric reconstruction of the aortic sinuses. Ao, Ascending aorta; $L V$, left ventricle; $R V$, right ventricle.

In the absence of annuloaortic ectasia, multiple interrupted horizontal mattress sutures of 4-0 polyester are passed from inside to outside the left ventricular outflow tract. These sutures are placed in a horizontal plane that corresponds to the lowest level of the leaflet attachments. In the presence of annuloaortic ectasia, these sutures are strategically placed to allow for correction of annuloaortic ectasia. On the right side of the ventriculoaortic junction, which is fibrous, the sutures are placed in a horizontal plane that corresponds to the lowest level of the leaflet attachments. On the left side of the ventriculoaortic junction, where the aortic valve is attached to ventricular muscle, the sutures follow the scalloped line of insertion of the right and left leaflets. Subsequently, all 3 aortic sinuses are reconstructed with continuous 5-0 polypropylene sutures (Prolene, Ethicon, Inc, Somerville, NJ) using an everting suturing technique (Fig 1, C). A collagen-impregnated tubular Dacron graft (Hemashield Woven Double Velour grafts, Meadox Medicals, Inc, Oakland, NJ) is selected whose
Table III. Latest postoperative Doppler echocardiographic study

\begin{tabular}{lc}
\hline & No. of patients \\
\hline Aortic regurgitation & \\
Grade 0 & 4 \\
Grade 1 & 5 \\
Grade 2 & 1 \\
Grade 3 & 0 \\
Grade 4 & 0 \\
Total & 10 \\
\hline
\end{tabular}

diameter is equal to the calculated external diameter of the ventriculoaortic junction (Fig $1, D$ ). The calculated external diameter of the ventriculoaortic junction is obtained by adding 4 to $6 \mathrm{~mm}$ (the thickness of the aortic wall) to the calculated internal diameter of the ventriculoaortic junction. In the presence of annuloaortic ectasia, a scalloped segment of the Dacron graft is excised to correspond to the commissure of the right and left leaflets. In this circumstance, care is taken to space the sutures in such a fashion as to correct the annuloaortic ectasia. The reconstructed aortic root is placed inside the Dacron graft and all sutures are tied outside.

Three stay sutures are put into the edge of the reconstructed aortic root above each commissure. A gentle pull on these sutures results in a restoration of the sinus height and a reduction of commissural diameter, with resultant coaptation of the leaflets. The edge of the reconstructed aortic root is subsequently sewn to the Dacron graft at the appropriate level with a continuous 4-0 or 5-0 Prolene suture. The coronary arteries are reimplanted in the Dacron graft with a continuous 5-0 Prolene suture. The upper end of the Dacron graft is then anastomosed to the ascending aorta with a continuous 4-0 Prolene suture. All aortic and coronary ostial anastomoses are secured externally by the application of fibrin glue. After release of the aortic crossclamp, aortic valve competence is determined by transesophageal Doppler echocardiography.

\section{Results}

The aortic crossclamping time was $61 \pm 18$ minutes. The duration of stay in the intensive care unit was 2.9 \pm 1.5 days. In-hospital mortality was 2 of $13(15.3 \%)$ patients; both deaths were related to complications of aortic dissection. In both patients the cardiopulmonary bypass time and aortic crossclamping time had been prolonged (up to 450 minutes and 220 minutes, respectively). The first patient, who had aortic root dilatation and additional type A aortic dissection, died on the first postoperative day of low cardiac output after aortic root reconstruction with replacement of the ascending aorta and aortic arch. Because of acute ischemic changes of the left ventricle resulting from the aortic dissection, the left anterior descending and circumflex coronary arteries had been bypassed with saphenous vein grafts; subsequent weaning from cardiopulmonary bypass had 
been difficult. The second patient, with type B aortic dissection, had been subjected to additional replacement of the aortic arch and descending aorta. He died on the sixth postoperative day of cerebral infarction.

Early postoperative echocardiographic studies demonstrated complete competence of the aortic valve in 6 patients. Five patients had AR grade 1, in 1 of whom the AR increased to grade 3 , necessitating aortic valve replacement 2 weeks after the operation. No other valverelated complications have occurred. At a mean followup of 2.1 years (range 4 to 44 months), the remaining 10 patients with aortic root reconstruction have had stable aortic valve function without evidence of progressive AR (median grade of AR was 1) or aortic stenosis (Table III, Fig. 2). All patients were free of symptoms and the mean New York Heart Association functional class was 1.2. None of the patients with successful aortic root reconstruction was prescribed anticoagulants.

\section{Discussion}

With the decline in prevalence of rheumatic fever, noninflammatory aortic root dilatation has become the most common cause of isolated AR. ${ }^{14}$ In patients with aneurysms of the aortic root and the ascending aorta and normal aortic valve anatomy, replacement of the ascending aorta and all 3 aortic sinuses with a Dacron graft as reported by David and coworkers ${ }^{5,6}$ and Sarsam and Yacoub ${ }^{7}$ potentially results in an excellent hemodynamic status with preservation of native aortic valve function and avoidance of long-term anticoagulation. Although this complication has not been reported as yet, the main concern relating to these techniques is the potential for mechanical damage to the aortic valve leaflets in the long term, which may be induced by their contact with the rough texture of the Dacron graft. The technique that we propose allows exact adaptation of the sizes of the sinotubular junction and (if dilated) the ventriculoaortic junction and symmetric reconstruction of the aortic sinuses, thus optimizing coaptation and maintaining structural integrity of the aortic valve leaflets. Prevention of contact of the aortic valve leaflets with the rough surface of the Dacron graft may result in superior preservation of aortic valve function and hence increased durability of the repair. As in other aortic root remodeling techniques, aortic sinus function may become somewhat restricted because of the implantation of the sinuses into a rigid cylinder. In our experience and that of others, dilatation of the sinotubular junction is most often associated with dilatation of the noncoronary sinus of Valsalva, followed by dilatation of the right and left sinuses, respectively. ${ }^{6}$ As a result of this, to achieve symmetric reconstruction of the aortic root, the excision of redundant tissue was most extensive in the noncoronary sinus, followed by that in the right and left sinuses, respectively. Reimplantation of the coronary ostia into the Dacron graft has not posed a problem in any of the 13 patients in this series. In 10 of the 11 surviving patients in this series, at a follow-up of up to 44 months, aortic valve function has been stable without any evidence of leaflet damage. Further follow-up is necessary to evaluate the longterm results of our modified technique.

In summary, our modified technique of aortic root reconstruction allowed preservation of the native aortic valve, preservation and symmetric reconstruction of the aortic sinuses, and adaptation of the diameters of the sinotubular and ventriculoaortic junctions, with resultant excellent aortic valve function. This modification prevents contact of the aortic valve leaflets with the Dacron graft. Further follow-up will clarify whether this technique enhances the durability of the valve repair.

\section{REFERENCES}

1. Bentall HH, DeBono A. A technique for complete replacement of the ascending aorta. Thorax 1968;23:338-9.

2. Gott VL, Pyeritz RE, Magovern GJ Jr, Cameron DE, McKusick VA. Surgical treatment of aneurysms of the ascending aorta in the Marfan syndrome: results of composite graft repair in 50 patients. N Engl J Med 1986;314:1070-4.

3. Kouchoukos NT, Marshall WG Jr, Wedige-Stecher TA. Elevenyear experience with composite graft replacement of the ascending aorta and aortic valve. J Thorac Cardiovasc Surg 1986;92: 691-705.

4. Mohr FW, Walther T, Baryalei M, Falk V, Schindewolf K, Scheidt A, et al. The SPV Toronto bioprosthesis: results in 100 patients. Ann Thorac Surg 1995;60:171-5.

5. David TE, Feindel CM. An aortic valve-sparing operation for patients with aortic incompetence and aneurysm of the ascending aorta. J Thorac Cardiovasc Surg 1992;103:617-22.

6. David TE, Feindel CM, Bos J. Repair of the aortic valve in patients with aortic insufficiency and aortic root aneurysm. J Thorac Cardiovasc Surg 1995;109:345-52.

7. Sarsam MAI, Yacoub M. Remodeling of the aortic valve anulus. J Thorac Cardiovasc Surg 1993;105:435-8.

8. Zimmerman J. The functional and surgical anatomy of the heart. Ann R Coll Surg Engl 1966;39:348-66.

9. Anderson RH, Devine WA, Ho SY, Smith A, McKay R. The myth of the aortic annulus: the anatomy of the subaortic outflow tract. Ann Thorac Surg 1991;52:640-6.

10. Sutton JP III, Ho SY, Anderson RH. The forgotten interleaflet triangles: a review of the surgical anatomy of the aortic valve. Ann Thorac Surg 1995;59:419-27.

11. Kunzelman KS, Grande KJ, David TE, Cochran RP, Verrier ED. Aortic root and valve relationship: impact on surgical repair. J Thorac Cardiovasc Surg 1994;107:162-70.

12. Reid K. The anatomy of the sinus of Valsalva. Thorax 1970;25: 79-85. 
13. Silver MA, Roberts WC. Detailed anatomy of the normally functioning aortic valve in hearts of normal and increased weight. Am J Cardiol 1985;55:454-61.

14. Olson LJ, Subramanian R, Edwards WD. Surgical pathology of pure aortic insufficiency: a study of 225 cases. Mayo Clin Proc $1984 ; 59: 835-41$

\section{Targeted}

The Journal of Thoracic and Cardiovascular Surgery gives you two tables of contents.

The condensed table of contents tells you at a glance what topics and authors are presented each month. The expanded table of contents gives you a brief abstract of each article. You select only those articles of most interest to you for further reading. 